\title{
Clinical outcomes following self-expanding metal stent placement for esophageal salvage
}

\author{
Diana H. Liang, MD, ${ }^{a}$ Eric Hwang, ${ }^{a}$ Leonora M. Meisenbach, RN, ACNP-BC, ${ }^{b}$ Min P. Kim, MD, FACS, ${ }^{a, b}$
}

Edward Y. Chan, MD, ${ }^{a}$ and Puja Gaur Khaitan, MD, FACS ${ }^{a, b}$

\begin{abstract}
Objectives: To assess the efficacy of self-expanding metal stents (SEMS) for esophageal salvage in patients who would otherwise require esophageal/conduit resection.
\end{abstract}

Methods: We performed a retrospective chart review of patients who had SEMS placed from January 2010 to December 2015. Patient demographics, esophageal stent characteristics, and outcomes were assessed in our patient cohort.

Results: Our study included a total of 83 patients. A total of 148 SEMS were placed, with 121 partially covered SEMS (pcSEMS) and 27 fully covered SEMS (cSEMS). A stent was placed more than once in $42.2 \%$ of the patients. Median duration of stent placement was 23 days. Indications for SEMS placement included esophageal leak after esophageal resection (45.8\%), spontaneous esophageal perforation $(22.9 \%)$, iatrogenic esophageal perforation $(20.5 \%)$, and esophageal obstruction $(9.6 \%)$. Complications from SEMS placement included 6 stent migrations and 1 esophageal perforation. Of the 6 stents that migrated, 2 were pcSEMS and 4 were cSEMS. In a patient who underwent stent placement for a stricture refractory to dilation, a perforation at the distal end was discovered 2 days after stent removal. The perforation healed after the second SEMS placement. Ultimately, 15 patients $(18.1 \%)$ had to undergo a subsequent esophagectomy or takedown of their conduit with an overall $81.9 \%$ salvage of native esophagus or conduits.

Conclusions: Our study demonstrates the successful use of SEMS in patients with anastomotic leaks, perforations, and recalcitrant strictures. (J Thorac Cardiovasc Surg 2017;154:1145-50)

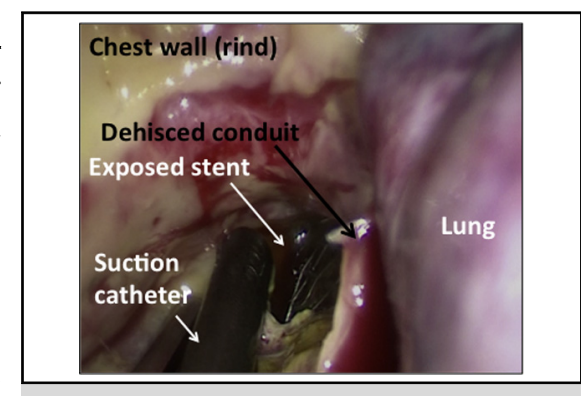

Salvage of a gastric conduit with the use of a partially covered esophageal stent. Right pleural space, apex: anastomotic dehiscence post esophagectomy successfully managed with an esophageal stent.

\section{Central Message}

Esophageal stenting is a viable option for patients with anastomotic leaks, esophageal perforations, and strictures refractory to dilation versus resection of gastric conduits or native esophagus.

\section{Perspective}

Stenting offers acceptable salvage outcomes in patients with complex esophageal and gastric pathology who otherwise would require major resection and diversion.

See Editorial Commentary page 1151.
Intrathoracic contamination from the esophagus, whether from an anastomotic leak after gastroesophageal resection, spontaneous rupture, or iatrogenic perforation, remains a life-threatening issue, with mortality rates as high as $60 \%$. $^{1}$ Historically, esophageal leak necessitated an aggressive surgical approach, including extensive debridement and possible esophageal diversion. Then, more conservative

\footnotetext{
From the ${ }^{\mathrm{a} D e p a r t m e n t}$ of Surgery and ${ }^{\mathrm{b}}$ Division of Thoracic Surgery, Weill Cornell Medical College, Houston Methodist Hospital, Houston, Tex.

Received for publication Nov 11, 2016; revisions received Feb 4, 2017; accepted for publication March 6, 2017; available ahead of print April 14, 2017

Address for reprints: Puja Gaur Khaitan, MD, FACS, Department of Surgery, Division of Thoracic Surgery, Weill Cornell Medical College, Houston Methodist Hospital, 6550 Fannin St, Suite 1661, Houston, TX 77030 (E-mail: pgaur@ houstonmethodist.org).

$0022-5223 / \$ 36.00$

Copyright (c) 2017 by The American Association for Thoracic Surgery http://dx.doi.org/10.1016/j.jtcvs.2017.03.051
}

surgical techniques of primary anastomotic repair with tissue reinforcement, as well as nonoperative management, were successfully adapted in select cases of intrathoracic contamination. $^{2}$ More recently, endoscopic placement of fully covered and partially covered stents has been explored and developed by thoracic surgeons to control esophageal leak (both cervical and thoracic) and perforation with acceptable results. ${ }^{3-5}$ Although the initial use of self-expanding metal stents (SEMS) was limited to palliation of tracheoesophageal fistulae from advanced

Scanning this $\mathrm{QR}$ code will take you to a supplemental video for the article.

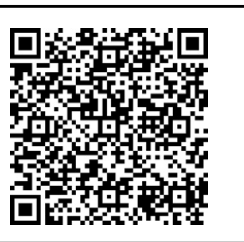




\section{Abbreviations and Acronyms}

cSEMS = covered self-expanding metal stents

pcSEMS $=$ partially covered self-expanding metal stents

SEMS $=$ self-expanding metal stents

malignancy, ${ }^{6-8}$ SEMS has since been used as an efficacious modality to control esophageal leak in the setting of anastomotic leak after esophagectomy, as well as iatrogenic or spontaneous esophageal perforations. ${ }^{1,4-6,9}$

In our institution, we have used covered SEMS (cSEMS) since 2007 , and have reported esophageal salvage in $77 \%$ of the patients with the use of cSEMS in the past. ${ }^{9}$ In 2010, we began using partially covered SEMS (pcSEMS) with exposed bare-metal mesh ends to promote tissue embedding. ${ }^{10}$ Thus, in this study, we evaluated the efficacy of SEMS in the management of esophageal leakage since the introduction of pcSEMS.

\section{METHODS}

Approval for this study was obtained from the institutional review board. From January 2010 to December 2015, 83 patients with gastroesophageal leakage underwent SEMS placement by the thoracic surgeons at our hospital as the primary means of controlling intrathoracic or intraabdominal contamination. Patients were consented for off-label use of SEMS based on previous success at our institution ${ }^{9}$ and other published studies, ${ }^{4,5}$ with the understanding that if they failed management with stent, they would need open surgical repair or even diversion. We retrospectively reviewed all patients in this cohort with the primary objective to evaluate the efficacy of SEMS in esophageal or conduit salvage. Patients undergoing esophageal stent placement for esophageal leak after esophagectomy, esophagojejunostomy, sleeve gastrectomy, foregut procedure, or spontaneous/iatrogenic perforation were all included in the study. We also included patients who had esophageal stricture refractory to dilation and had a stent placed as a way to salvage their native esophagus. However, patients with malignant tracheaesophageal fistula $(\mathrm{n}=6)$ or malignant mass causing esophageal obstruction $(n=4)$ who had stents placed for palliative intent were excluded from the study, as they were never deemed to be candidates for resection. Reviewed data included patient demographics, SEMS characteristics, postoperative stent-specific complications, and clinical outcomes. Although failure was defined as no change in the leak size or clinical signs of ongoing infection, successful salvage was defined by number of patients who did not have to undergo esophageal resection or conduit takedown.

Statistical analyses were performed by using Microsoft Excel (Microsoft, Redmond, Wash). Data are reported as mean $\pm 95 \%$ confidence intervals or as median (minimum-maximum) values.

\section{RESULTS}

\section{Patient Demographics}

From January 2010 to December 2015, 83 patients were identified to have gastroesophageal leakage controlled with endoscopic placement of SEMS that met our inclusion criteria (Table 1). The median age was 63 years. In this study, there were $56(67.5 \%)$ men and $27(32.5 \%)$ women. The comorbidities are detailed in Table 1 .
TABLE 1. Patient demographics $(n=83)$

\begin{tabular}{lc}
\hline Age, $\mathrm{y}$, median (range) & $63(31-85)$ \\
Gender, $\mathrm{n}(\%)$ & \\
Male & $56(67.5)$ \\
Female & $27(32.5)$ \\
Comorbidities, $\mathrm{n}(\%)$ & \\
Diabetes & $15(18.1)$ \\
Hypertension & $52(62.7)$ \\
Coronary artery disease & $22(26.5)$ \\
Chronic obstructive pulmonary disease & $8(9.6)$ \\
Chronic kidney disease & $7(8.4)$ \\
Cerebrovascular accident & $5(6.0)$ \\
Smoking history & $39(47.0)$ \\
Malignancy, $\mathrm{n}(\%)$ & $27(32.5)$ \\
Adenocarcinoma & 24 \\
Squamous cell carcinoma & 1 \\
Others & 2 \\
History of radiation therapy, $\mathrm{n}(\%)$ & $24(28.9)$ \\
History of chemotherapy, $\mathrm{n}(\%)$ & $22(26.5)$ \\
Indications, $\mathrm{n}(\%)$ & \\
Iatrogenic perforation & $17(20.5)$ \\
Spontaneous perforation & $19(22.9)$ \\
Esophageal leak after surgery & $38(45.8)$ \\
Obstruction & $8(9.6)$ \\
Severe esophageal caustic injury & $1(1.2)$ \\
\hline
\end{tabular}

A significant proportion of our patients had risk factors predisposing them to gastroesophageal leakage, particularly anastomotic leakage after esophagectomy. Of 27 patients who underwent an esophagectomy for malignancy or dysplasia, 25 patients received neoadjuvant chemoradiation, 1 patient had history of radiation therapy only, and 1 patient had history of chemotherapy alone. One patient underwent esophagectomy for a benign stricture.

Indications for endoscopic SEMS placements were iatrogenic esophageal perforation in $17(20.5 \%)$ patients, spontaneous esophageal rupture in $19(22.9 \%)$ patients, anastomotic leak after gastroesophageal resection in $38(45.8 \%)$, esophageal obstruction in $8(9.6 \%)$, and severe esophageal caustic injury from ingestion in 1 patient. Of 38 patients who had anastomotic leak after surgical resection, 22 patients had undergone Ivor Lewis esophagectomy, 5 patients 3-hole esophagectomy, 1 patient transhiatal esophagectomy, 1 patient esophagectomy with colonic interposition, 1 patient with delayed esophageal reconstruction after staged esophagectomy, 3 patients esophagojejunostomy, 3 sleeve gastrectomy, and 2 Heller myotomy with fundoplication (Table 2).

\section{Stent Characteristics}

In 83 patients, a total of 148 stents were placed, which included 121 pcSEMS and 27 cSEMS (Table 3). Forty-eight $(57.8 \%)$ patients required only 1 SEMS placement, with no replacement with additional SEMS. Seventeen patients required 2 consecutive SEMS placements, 11 patients needed 3 stents placed, and 7 patients needed 4 or more stents. During this time, we 
TABLE 2. Patients who had esophageal leak after surgery $(n=38)$

\begin{tabular}{lr}
\hline \multicolumn{1}{c}{ Types of surgery } & $\mathbf{n}$ \\
\hline Ivor Lewis esophagectomy & 22 \\
Transhiatal esophagectomy & 1 \\
3-hole esophagectomy & 5 \\
Esophagojejunostomy & 3 \\
Sleeve gastrectomy & 3 \\
Heller myotomy with fundoplication & 2 \\
Esophagectomy with colonic interposition & 1 \\
Delayed esophageal reconstruction & 1 \\
\hline
\end{tabular}

used only Ultraflex and WallFlex stents (cSEMS and pcSEMS, respectively) from Boston Scientific (Marlborough, Mass). All SEMS were placed endoscopically under fluoroscopic guidance by thoracic surgeons at our institution. The surgeon used individualized clinical judgment to determine the choices of either cSEMS or pcSEMS placement. On-table contrast studies were performed to evaluate for successful placement of SEMS to exclude the area of gastroesophageal leakage. To prevent migration, 72 SEMS were fixed with umbilical tape as described previously, ${ }^{11}$ and 12 were fixed with the OverStitch Endoscopic Suturing System (Apollo, Austin, Tex) (Video 1). Whenever umbilical tape was used to secure the stent, it was cut 4 days after the SEMS placement to allow sufficient time for tissue embedding. However, the endoscopic suture was removed only at the time of stent

TABLE 3. Esophageal stent characteristics

\begin{tabular}{|c|c|}
\hline Type of stent $(\mathrm{n}=148)^{*}$ & $\mathbf{n}$ \\
\hline Partially covered self-expanding metal stent & 121 \\
\hline Fully covered self-expanding metal stent & 27 \\
\hline \multicolumn{2}{|l|}{ Number of stent placements } \\
\hline 1 stent & 48 \\
\hline 2 stents & 17 \\
\hline 3 stents & 11 \\
\hline 4 or more stents & 7 \\
\hline \multicolumn{2}{|l|}{ Stent duration $(\mathrm{n}=135) \dagger$} \\
\hline Days, median (range) & $23(0-57)$ \\
\hline Days, average $(95 \%$ confidence interval $)$ & $21.8(20,23.6)$ \\
\hline \multicolumn{2}{|l|}{ Complications } \\
\hline Migration & 6 \\
\hline Perforation & 1 \\
\hline \multicolumn{2}{|l|}{ Fixation technique for migrated stents $(n=6)$} \\
\hline Umbilical tape & 4 \\
\hline Apollo stitch & 0 \\
\hline Not fixed & 2 \\
\hline \multicolumn{2}{|l|}{ Type of stent for migrated stents $(n=6) \ddagger$} \\
\hline Partially covered self-expanding metal stent & 2 \\
\hline Fully covered self-expanding metal stent & 4 \\
\hline
\end{tabular}

\section{Clinical outcomes following self- expanding metal stent placement for esophageal salvage}

\author{
D. H. Liang, E. Hwang, L. M. Meisenbach, \\ M. P. Kim, E. Y. Chan, P. G. Khaitan
}

VIDEO 1. The decision-making process to select patients appropriate for esophageal stent placement and 2 different modes of anchoring a stent in place to reduce the risk of stent migration. Video available at: http:// www.jtcvsonline.org/article/S0022-5223(17)30561-5/addons.

removal. Patients with intrathoracic sepsis were managed with tube thoracostomy, video-assisted thoracoscopic decortication, or thoracotomy. Most SEMS were removed in 3 to 4 weeks in the operating room with reevaluation of leak with an on-table esophagram. Patients with persistent gastroesophageal leakage underwent repeat SEMS placement with reevaluation every 3 to 4 weeks until the site healed. Patients who failed conservative management with SEMS or developed deteriorating clinical status ultimately underwent esophageal diversion.

\section{Postoperative Events}

There were a total of 6 stent migrations ( 2 pcSEMS, 4 cSEMS), 4 of which were bridled with umbilical tape and 2 that were not fixed, resulting in a $5.6 \%$ migration incidence for SEMS fixed with umbilical tape. Interestingly, none of the 12 stents fixed with the endoluminal suturing device resulted in migration and of the stents that were not fixed, only 2 migrated, resulting in a $3.1 \%$ migration rate where both were cSEMS. When further divided, this gave us a $14.8 \%$ migration incidence for cSEMS and $1.7 \%$ for pcSEMS. Median duration of SEMS placement was 23 days, ranging 0 to 57 days. The patient with 0 -day duration was a patient who had undergone a Nissen fundoplication 2 weeks earlier, who presented to our institution in extremis with severe metabolic acidosis and cardiovascular instability. Endoscopy revealed an esophageal perforation just proximal to the gastroesophageal junction with an extremely dilated and ischemic stomach. Therefore, pcSEMS was placed with the intent to emergently control the source of sepsis; however, the patient died of cardiac arrest soon after in the intensive care unit.

One of our patients who had SEMS placement for benign esophageal stricture refractory to multiple endoscopic dilations had pneumoperitoneum discovered 2 days after the stent removal secondary to a perforation in the distal esophagus just distal to the distal end of the stent. She underwent repeat SEMS placement along with gastrostomy tube placement at the time of laparoscopic abdominal 
TABLE 4. Stent complications based on underlying indication for initial stent placement

\begin{tabular}{lccccc}
\hline & $\begin{array}{c}\text { Iatrogenic } \\
\text { perforation }(\mathbf{n}=\mathbf{1 7})\end{array}$ & $\begin{array}{c}\text { Spontaneous } \\
\text { perforation }(\mathbf{n}=\mathbf{1 9})\end{array}$ & $\begin{array}{c}\text { Postsurgical } \\
\text { leak }(\mathbf{n}=\mathbf{3 8})\end{array}$ & $\begin{array}{c}\text { Obstruction/stricture } \\
(\mathbf{n}=\mathbf{8})\end{array}$ & $\begin{array}{c}\text { Caustic injury } \\
(\mathbf{n}=\mathbf{1})\end{array}$ \\
\hline Migration & 1 & 0 & 4 & 1 & 0 \\
Perforation & 0 & 0 & 0 & 1 & 0 \\
Concomitant drainage procedure & 6 & 12 & 13 & 0 & 1 \\
Unable to salvage & 2 & 4 & 8 & 0 \\
\hline
\end{tabular}

exploration. The second SEMS was removed 25 days later, with resolution of the perforation confirmed via on-table esophagram.

A summary of post-stent complications, including migration, perforation, and inability to salvage the esophagus, is briefly summarized in Table 4 . The highest incidence of stent migration was noted to be in the postsurgical group of patients, with lowest incidence in the spontaneous perforation group.

\section{Clinical Outcomes}

The esophageal/conduit salvage success was $81.9 \%$. There were 15 patients who failed endoscopic management with SEMS for gastroesophageal leak and ultimately required conduit takedown or esophagectomy (Table 5). Of these 15 patients, $9(60 \%)$ patients underwent SEMS placement more than once in an attempt to salvage the esophagus or conduit, and $6(40 \%)$ underwent an additional drainage operation in addition to SEMS placement. The median number of days from SEMS placement to definitive resection was 24 days, ranging from 1 to 75 days. One patient with a history of intermittent dysphagia required esophagectomy only 1 day after SEMS placement. She had presented to our institution after delayed diagnosis of spontaneous esophageal perforation at another hospital. Tube thoracostomy and pcSEMS were placed emergently as a temporizing source control measure during

TABLE 5. Patients unable to salvage esophagus/conduit/stomach $(\mathbf{n}=\mathbf{1 5})$

Indications for stent placement that failed to salvage esophagus

Iatrogenic perforation 2

Spontaneous perforation

Esophageal leak after surgery

Obstruction (stricture)

Total number of stents

Patients requiring stent more than once

Patients requiring additional drainage at the time of stent placement

Days from stent to definitive resection*

Days, median (range)

$24(1-75)$

Days, average ( $95 \%$ confidence interval)

$33(19,47)$

* One patient had esophagectomy 1 day after stent placement. This patient had stent placement as a temporizing measure during resuscitation. Purpose of this stent was not for attempt at esophageal salvage. resuscitation before definitive esophagectomy with proximal esophageal diversion. One year later, she successfully underwent reconstruction with a jejunal conduit to reestablish esophageal continuity.

\section{DISCUSSION}

In the past decade, upper gastrointestinal stents, initially developed as rigid cylinders to palliate malignant obstructions, have been modified to flexible and self-expandable forms to widen applicability and improve efficacy and safety. ${ }^{12}$ Metal stents, characterized by their greater degree of flexibility and their ability to generate high radial forces for stent patency and position, are available as uncovered, partially covered, or fully covered. ${ }^{12,13}$ Compared with plastic stents with previously reported migration as high as $35 \%,{ }^{6}$ metal stents have been associated with lower incidence of migration and lower incidence of stent failure requiring reintervention. ${ }^{4,5,12-14}$ Among the metal stents, uncovered SEMS are associated with the highest risk of tissue ingrowth leading to stent occlusion and with difficulty in stent removal, despite their lower rates of migration. ${ }^{12,13}$ Therefore, we have advocated the use of either cSEMS or pcSEMS. In a previous study from our institution, 2 episodes $(6.7 \%)$ of stent migration occurred among 30 patients with cSEMS placements. ${ }^{9}$ In another study, stent migration was reported to be significantly higher with cSEMS $(37.5 \%)$ versus pcSEMS $(9.1 \%) .{ }^{10} \mathrm{~A}$ systematic review has shown similar trend with $26 \%$ migration with cSEMS and $12 \%$ with pcSEMS reported from other institutions. ${ }^{15}$ Since the publication of these studies, our group has published a fixation method, bridling of SEMS with umbilical tape.$^{11}$ More recently, we have adopted the use of an endoluminal suturing device (Apollo) for stent fixation (although this is not approved by the Food and Drug Administration for this purpose). During the initial employment of the bridling method, we saw complete elimination of stent migration. However, with a much larger sample size, we report 5.6\% migration of stents fixed with umbilical tape, no migration of 12 stents fixed with the endoluminal suturing device, and 3.1\% migration of stents that were not fixed with either method. Results in Table 4 indicate a higher incidence of stent migration in the postsurgical leak cohort and the lowest incidence in the spontaneous perforation cohort; however, 


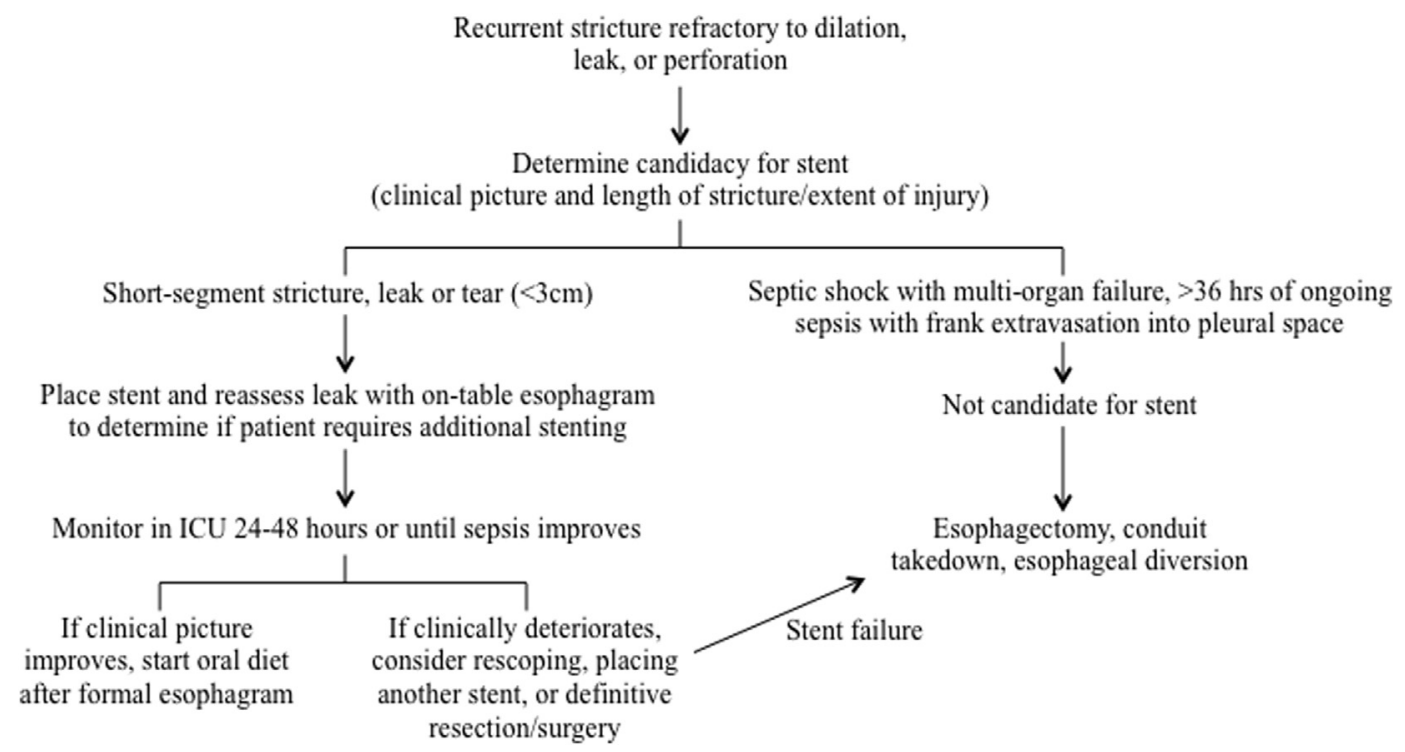

FIGURE 1. Proposed algorithm for esophageal stent placement for patients with esophageal strictures, leaks, or perforations. ICU, Intensive care unit.

these results are difficult to interpret, as the subsets are too small to compare.

With improvements in the design of stents, gastroenterologists and thoracic surgeons have broadened the indications for stent placement to encompass benign strictures, perforations, and anastomotic leakage after gastroesophageal resections. ${ }^{12}$ Although the palliation of inoperable malignant obstruction remains the most common indication of esophageal stenting in the literature, ${ }^{11}$ patients with any gastroesophageal leak also can be successfully managed with endoscopic intervention and subsequent salvage of the native organs. With our proposed algorithm (Figure 1), we report a clinical success with $81.9 \%$ of our patients at a single institution, defined as resolution of gastric/esophageal leak or obstruction secondary to stricture without either takedown of the esophageal conduit, esophagectomy, or gastrectomy. Similar to our findings, a systematic review of 25 articles by van Boeckel and colleagues ${ }^{15}$ that included 267 patients $(51 \%$ anastomotic leak, $25 \%$ iatrogenic perforation, $17 \%$ spontaneous perforation) had reported $85 \%$ success with endoscopic treatment of anastomotic leak with esophageal stent. ${ }^{1}$

Potential complications from esophageal stent insertion include perforation, erosion, bronchoesophageal fistula, and recurrent obstruction due to tumor ingrowth. As in any iatrogenic esophageal perforation, stent-related perforation can be a life-threatening event. There are various reports of complications in the literature secondary to stent, such as perforation into the trachea ${ }^{16}$ and esophageal necrosis at the proximal end of stent, ${ }^{17}$ as well as enlargement of the esophageal leak $^{4}$ and extension of anastomotic dehiscence. ${ }^{18}$ However, the only esophageal perforation we experienced in our study was a result of the initial stent with no distal obstruction that was diagnosed in a timely manner before clinical deterioration, which resolved after stent exchange. Our one listed mortality was from a patient who had presented to us in extremis and was not directly related to the stent itself.

\section{Study Limitations}

Our study has the limitations inherent to all retrospective outcome studies. Our study consisted of a small and heterogeneous group of patients with an uncommon clinical problem. It is not meaningful to compare leaks and perforations with a stricture population. However, because the main purpose of our study was to demonstrate successful salvage, we decided to include refractory strictures as candidates for stent, because if these patients failed conservative therapy with serial dilations, they would be eventually counseled to undergo an esophagectomy or risk an iatrogenic perforation with repeated dilations and trauma. Although a comparison group of patients without stent therapy would be beneficial to demonstrate the efficacy of stent use, we cannot provide such a group because stent therapy has primarily become the standard of care at our institution for this patient population over the past 10 years. Furthermore, there is a selection bias inherent to this study. Because a septic, clinically unstable patient would not have been considered for a stent and may have indeed undergone a diversion, and because this study is focused on those patients who were initially treated with a stent, it has automatically excluded those patients who were not candidates for stenting to begin with; this may have resulted in a falsely elevated salvage rate. And finally, the study is limited by our inpatient data only (as we did not have outpatient follow-up data or information on death from other causes) and the use of covered and partially covered stents without any strict guidelines. 
Although the judgment to place cSEMS versus pcSEMS was dependent on the surgeon's discretion, some of the criteria used at our institution to place covered stents were as follows: cSEMS were chosen if the leak, perforation, or stricture site was very high and close to the upper esophageal sphincter to avoid the uncovered portion of the stent to cause neck pain and persisting globus sensation. Additionally, patients with friable esophagus whereby the uncovered portion of the stent could result in granulation tissue formation that could then cause significant injury during stent removal were considered an ideal group of patients for cSEMS; these were concomitantly anchored, as covered stents are anecdotally at higher risk for migration. Despite these limitations, our study is one of the largest case series currently available, and is unique in that we included all patients with gastroesophageal leakage who underwent endoscopic management and all patients with stricture who had failed repeated dilations and would have otherwise needed an esophagectomy.

Given our experience and outcome, we have proposed an algorithm (Figure 1) on how to evaluate patients for stent placement versus definitive repair or even diversion. Furthermore, we are not suggesting that all spontaneous perforations be handled with stents; indeed, we recommend that if a patient has a spontaneous perforation with frank extravasation into the pleural space or gross contamination of the mediastinum, a thoracoscopic or even open washout is warranted, along with a primary repair of the perforation site with a muscle flap or even diversion. However, if a patient suffers from rather limited perforation or incidental pneumomediastinum whereby the esophagram demonstrates the contrast to be walled off with little or no spillage, a stent can be considered as long as the patient is hemodynamically stable.

\section{CONCLUSIONS}

Based on our study presented here, we recommend the use of SEMS in a carefully selected patient population, with an acceptable salvage success. We have hereby proposed an algorithm highlighting the criteria to decide when to stent patients and demonstrated 2 techniques that we use at our institution to anchor stents to decrease the incidence of migration (Video 1).

\section{Conflict of Interest Statement}

Authors have nothing to disclose with regard to commercial support.

\section{References}

1. Schaheen L, Blackmon SH, Nason KS. Optimal approach to the management of intrathoracic esophageal leak following esophagectomy: a systematic review. Am J Surg. 2014;208:536-43.

2. Crestanello JA, Deschamps C, Cassivi SD, Nichols FC, Allen MS, Schleck C, et al. Selective management of intrathoracic anastomotic leak after esophagectomy. J Thorac Cardiovasc Surg. 2005;129:254-60.

3. Chak A, Singh R, Linden PA. Covered stents for the treatment of life-threatening cervical esophageal anastomotic leaks. J Thorac Cardiovasc Surg. 2011;141: $843-4$

4. D'Cunha J, Rueth NM, Groth SS, Maddaus MA, Andrade RS. Esophageal stents for anastomotic leaks and perforations. J Thorac Cardiovasc Surg. 2011;142: 39-46.e1.

5. Freeman RK, Herrera A, Ascioti AJ, Dake M, Mahidhara RS. A propensitymatched comparison of cost and outcomes after esophageal stent placement or primary surgical repair for iatrogenic esophageal perforation. $J$ Thorac Cardiovasc Surg. 2015;149:1550-5.

6. Dai Y, Chopra SS, Kneif S, Hunerbein M. Management of esophageal anastomotic leaks, perforations, and fistulae with self-expanding plastic stents. J Thorac Cardiovasc Surg. 2011;141:1213-7.

7. Brown TH, Nicholson DA, Irving MH, Bancewicz J. Use of a selfexpanding metal stent for oesophagogastric fistulation. $\mathrm{Br}$ J Surg. 1995; 82:663-4.

8. May A, Ell C. Palliative treatment of malignant esophagorespiratory fistulas with Gianturco-Z stents. A prospective clinical trial and review of the literature on covered metal stents. Am J Gastroenterol. 1998;93:532-5.

9. David EA, Kim MP, Blackmon SH. Esophageal salvage with removable covered self-expanding metal stents in the setting of intrathoracic esophageal leakage. Am J Surg. 2011;202:796-801; discussion 801.

10. Seven G, Irani S, Ross AS, Gan SI, Gluck M, Low D, et al. Partially versus fully covered self-expanding metal stents for benign and malignant esophageal conditions: a single center experience. Surg Endosc. 2013;27: 2185-92.

11. Lyons CD, Kim MP, Blackmon SH. A novel fixation procedure to eliminate covered self-expanding metal stent migration. Ann Thorac Surg. 2012;94: 1748-50.

12. Kang HW, Kim SG. Upper gastrointestinal stent insertion in malignant and benign disorders. Clin Endosc. 2015;48:187-93.

13. Kochar R, Shah N. Enteral stents: from esophagus to colon. Gastrointest Endosc. 2013;78:913-8.

14. Freeman RK, Ascioti AJ. Esophageal stent placement for the treatment of perforation, fistula, or anastomotic leak. Semin Thorac Cardiovasc Surg. 2011;23: 154-8.

15. van Boeckel PG, Sijbring A, Vleggaar FP, Siersema PD. Systematic review: temporary stent placement for benign rupture or anastomotic leak of the oesophagus. Aliment Pharmacol Ther. 2011;33:1292-301.

16. Feith M, Gillen S, Schuster T, Theisen J, Friess H, Gertler R. Healing occurs in most patients that receive endoscopic stents for anastomotic leakage; dislocation remains a problem. Clin Gastroenterol Hepatol. 2011;9:202-10.

17. Brangewitz M, Voigtlander T, Helfritz FA, Lankisch TO, Winkler M, Klempnauer J, et al. Endoscopic closure of esophageal intrathoracic leaks: stent versus endoscopic vacuum-assisted closure, a retrospective analysis. Endoscopy. $2013 ; 45: 433-8$.

18. Schweigert M, Solymosi N, Dubecz A, Stadlhuber RJ, Muschweck H, Ofner D, et al. Endoscopic stent insertion for anastomotic leakage following oesophagectomy. Ann R Coll Surg Engl. 2013;95:43-7.

Key Words: esophageal stent, stent complications, techniques to anchor an esophageal stent, esophageal/conduit salvage, algorithm 